\title{
La política global del Banco Mundial hacia las políticas sociales y la cuestión social (1990-2018)
}

\section{The World Bank's global policy towards social policies and the social question (1990-2018)}

\author{
Emiliano Fernández \\ emilianofernandez33@gmail.com \\ Núcleo de Investigación Crítica sobre Sociedad y \\ Estado. Universidad Nacional del Centro de la \\ Provincia de Buenos Aires, Argentina \\ CONICET, Argentina
}

Recepción: 22 Febrero 2021

Aprobación: 15 Julio 2021

Publicación: 02 Agosto 2021

Cita sugerida: Fernández, E. (2021). La política global del Banco Mundial hacia las políticas sociales y la cuestión social (1990-2018). Cuestiones de Sociología, 25, e126. https://doi.org/10.24215/23468904e126
Resumen: El objetivo de este trabajo es caracterizar la política del Banco Mundial hacia las políticas sociales y la cuestión social durante el período que se extiende desde la década de los años noventa hasta la actualidad. En particular, buscamos fundamentar la siguiente idea: durante las últimas dos décadas se consolida la orientación e intervención hacia las políticas sociales y la cuestión social que el BM articuló en la década de los años noventa. Se trata de la consolidación de una orientación política que busca fortalecer las políticas sociales y, en particular, las políticas asistenciales como instrumentos de contención política de la masa de población pobre resultante de la acumulación capitalista, al mismo tiempo que procura sostener sus atributos productivos mínimos para los distintos requerimientos dicha acumulación. Para ello, metodológicamente, nos valdremos de documentos provistos por el propio organismo, como también de series estadísticas provistas también por ese organismo y por otros organismos internacionales.

Palabras clave: Banco Mundial, Políticas Sociales, Política Asistencial, Cuestión Social.

Abstract: The aim of this paper is to characterize the World Bank's policy towards social policies and the social question during the period from the 1990 s to the present. In particular, we seek argue the following idea: during the last two decades the orientation and intervention towards social policies and the social question that the World Bank articulated in the 1990s is consolidated. This is the consolidation of a political orientation that seeks to strengthen social policies and, in particular, assistance policies as instruments of political containment of the mass of poor people resulting from capitalist accumulation, at the same time as it seeks to sustain its minimum productive attributes for the various requirements of such accumulation. For this, methodologically, we will use documents provided by the organization itself, as well as statistical series.

Keywords: World Bank, Social Policy, Assistance Policy, Question Social. 


\section{1-Introducción ${ }^{1}$}

Según uno de los últimos informes del Overseas Development Institute (ODI, 2018), el Banco Mundial es el banco de desarrollo multilateral que posee mayor cantidad de miembros con un total de 189 países; la mayor cantidad de oficinas, con un total de 106 a lo largo del mundo; la mayor planta de personal, con 11 mil personas empleadas, y el doble de producción de documentos que todos los demás bancos en conjunto ( 22 mil frente a 12 mil documentos). Además, es el organismo financiero para el desarrollo que posee mayor capital suscripto por sus accionistas, el segundo con más reservas detrás del Banco de Inversión Europeo (EIB, por sus siglas en inglés); y en conjunto el BIRF (Banco Internacional para la Reconstrucción y Fomento) y la AIF (Asociación Internacional de Fomento), ambos organismos que conforman el Grupo Banco Mundial, concentran más de la mitad del total de la cartera de préstamos de todo el sistema de organismos financieros oficiales. ${ }^{2}$ Es decir, el Banco Mundial (BM), en tanto organismo internacional financiero, político e intelectual, posee centralidad en el sistema financiero y político internacional. De aquí la importancia de su estudio en profundidad. $^{3}$

El BM ha sido estudiado en nuestro medio desde numerosas aristas, entre ellas su relación con las políticas sociales y la cuestión social (véanse: Álvarez Leguizamón, 2001; Chiara y Di Virgilio, 2005; Coraggio, 1994; Corbalán, 2002; Grassi, Hintze y Neufeld, 1994; Murillo, 2006). No obstante, quizás a excepción del trabajo de Carcedo (2017), la mayoría de los análisis se concentra en la década de los años noventa, siendo casi inexistente producción que analice las últimas dos décadas del siglo XXI. Mientras algunos trabajos de la literatura internacional avanzaron en explorar la primera década del actual siglo (Babb, 2009; Hall, 2007; Mendes Pereira, 2017; Ruckert, 2010).

El objetivo de este artículo es caracterizar la política global del Banco Mundial hacia las políticas sociales y la cuestión social durante el período que se extiende desde la década de los años noventa hasta la actualidad. En particular, buscamos fundamentar la siguiente idea: durante las últimas dos décadas se consolida la orientación e intervención hacia las politicas sociales y la cuestión social que el BM articuló en la década de los años noventa. Se trata de la consolidación de una orientación política que busca fortalecer las políticas sociales y, en particular, las políticas asistenciales como instrumentos de contención política de la masa de población pobre resultante de la acumulación capitalista, al mismo tiempo que procura sostener sus atributos productivos mínimos para los distintos requerimientos del capital en el mercado de trabajo. Lo hace actualizando la tradición tutelar y controladora que matriza, en buena parte del mundo, las políticas sociales y, en particular, las políticas asistenciales (Garland, 2018; Mallardi, 2016).

En términos metodológicos, el artículo descansa en el análisis documental a partir de los documentos oficiales del organismo internacional, centralmente sus informes anuales, y en el análisis estadístico descriptivo a partir de la información proveniente del mismo organismo sobre su cartera de créditos y de otros organismos internacionales (OCDE, OIT, FMI). La delimitación temporal del artículo es el período 1990-2018, que permite abarcar el tramo en que el BM comienza con una política neoliberal más enfática hacia las políticas sociales y la 
configuración que adquiere esa política en las dos décadas del presente siglo antes de la actual crisis mundial disparada por la pandemia del Covid-19.

Organizamos el trabajo, luego de la introducción, en tres partes: el segundo apartado plantea una caracterización de la política general del Banco Mundial, que se inicia con una breve referencia a su trayectoria histórica y luego enfatiza en sus principales lineamientos políticos y el perfil de su política técnico-financiera en las últimas tres décadas; el tercer apartado avanza en una caracterización de la política del Banco hacia las políticas sociales y la cuestión social, revisando su surgimiento y su configuración a partir de la década de los años noventa, y resaltando la forma que adquiere durante las últimas dos décadas del presente siglo; el cuarto apartado establece conclusiones a partir de recuperar los aportes desarrollados en el trabajo.

\section{2 - El Banco Mundial en la globalización neoliberal}

Si bien el Banco Mundial es fruto directo del orden de posguerra articulado alrededor del impulso estadounidense, concebido inicialmente para apuntalar el derruido mapa europeo tras la guerra y alejar la amenaza comunista, su papel a lo largo de la historia rebasó esa marca de origen (Babb, 2009). Durante sus primeros años de vida se orientó a financiar infraestructura en los países atrasados y, en los albores de la década del sesenta, también políticas hacia la educación, salud y el ámbito rural. ${ }^{4} Y$, como veremos más adelante, la bandera de la lucha contra la pobreza es arriada en los tempranos años setenta. El período de reestructuración capitalista abierto tras la crisis del orden de posguerra hacia fines de los sesenta y principios de los setenta fue el marco en el que la organización internacional, en compás con el FMI, se reorientó en sintonía con la ofensiva neoconservadora. De sostener una orientación destinada fundamentalmente al financiamiento de infraestructura y al sector agrícola bajo un paradigma modernizador/desarrollista pasa, sin dejar de financiar lo anterior, a enfatizar en préstamos para el llamado ajuste estructural de los países endeudados y a ser uno de los principales agentes internacionales impulsores del paradigma neoliberal. Esta reorientación comienza a construirse hacia finales de la década de los setenta. En mayo de 1979, en una iniciativa para recuperar la demanda de créditos y lograr más accionistas en un contexto de cuestionamiento de las contribuciones norteamericanas por parte de los republicanos, el Banco lanza los préstamos de ajuste estructural. Aunque en un principio estaban orientados a apoyar la balanza de pagos de los países que dependían de la importación de petróleo, luego se generalizaron aportando a la reestructuración capitalista en curso (Bonnet, 2001; Cleaver, 1989; Holloway, 1994). Los préstamos de ajuste estructural se orientaron a la reestructuración del estado y la economía del país prestatario, impulsando la liberalización del comercio, la alineación de precios nacionales respecto al mercado internacional, la reducción en las barreras tarifarias, la devaluación de la moneda, medidas para atraer inversión extranjera y esfuerzos para promover la especialización y ampliar las exportaciones, en especial las mercancías agrícolas (Mendes Pereira, 2012). En la administración pública los ajustes tenían el propósito central de reducir el déficit fiscal, en especial a través de recortes en el gasto de personal y administración, reducciones en los subsidios al consumo, ahorros en el costo per cápita de los programas públicos 
con el objeto de ampliar la cobertura y reorientación de la política social para enfocar los gastos en atención primaria de la salud y en educación básica hacia poblaciones en condiciones de "pobreza absoluta". De 1978 a 1982, todas estas medidas figuraron como recomendaciones de política sectorial o económica en los más importantes informes del Banco (Mendes Pereira, 2012). Como señala Babb (2013), estos préstamos fueron marginales durante algunos años por la desconfianza de parte del gobierno de Reagan y un cuestionamiento más general a la eficiencia de las Instituciones Financieras Internacionales (IFI). Esto se revierte con el Plan Baker, en el segundo mandato de Reagan. En efecto, hacia mediados de los ochenta el Plan Baker impulsa los préstamos de ajuste estructural y, en el mismo proceso, reposiciona al Banco Mundial -como también al BIDen el sistema internacional. Así, los créditos de ajuste estructural -marginales antes- ganan peso en la cartera del Banco Mundial. Hacia fines de la década de los 80, los préstamos de ajuste estructural representaban entre el 20 y el 30 $\%$ de los desembolsos anuales del Banco. Y, hacia fines de los años noventa, el Informe Anual de 1998 del BM señalaba que los préstamos para ajuste estructural equivalían al $39 \%$ del total de la cartera y los de ajuste sectorial al $8 \%$ del total. De este modo, el BM formó parte de la ofensiva del capital contra el trabajo a escala mundial, aportando a construir el 'paradigma político transnacional neoliberal' en términos de un conjunto de ideas y prácticas de políticas públicas con sustento teórico, pero al mismo tiempo basado en la experiencia de prácticas concretas de los estados y las propias organizaciones internacionales (Babb, 2013).

No obstante, hacia finales de la última década del siglo veinte, las resistencias sociales a la disciplina monetaria en diferentes países fuerzan al Banco Mundial -como a otras IFI- a reajustar su orientación e intervención. En abril de 1998, durante la Cumbre de las Américas, James Wolfensohn, presidente por entonces del Banco Mundial, declaró que el Consenso de Washington estaba terminado. Se trataba más bien de una reorientación hacia dentro mismo del paradigma neoliberal. ${ }^{5}$ En lo fundamental se plantea la insuficiencia de las reformas de mercado para lograr el objetivo del crecimiento económico y todas sus bondades. En esta perspectiva, las llamadas "reformas de primera generación" (basadas en la liberalización comercial y financiera, las privatizaciones y la desregulación del mercado laboral) debían ser complementadas por reformas de "segunda generación”. Estas debían apuntar a la modernización del estado, o sea, a la racionalización de los procedimientos en la administración pública. En el documento Más allá del Consenso de Washington. La Hora de la Reforma Institucional, publicado en el año 1998 por el mismo Banco Mundial, se cristaliza conceptualmente con mayor claridad el imperativo de la "modernización del estado". Aquí las reformas de segunda generación son presentadas como propiciadoras de la calidad institucional, que garantizarían "tasas de crecimiento sostenibles y una reducción más significativa de la pobreza”. Las reformas institucionales promovidas por el Banco están asentadas teóricamente en lo que se denomina el paradigma de la Nueva Gestión Pública y en el neoinstitucionalismo. ${ }^{6}$ Este lineamiento sobrevive al ciclo de impugnaciones al neoliberalismo dado en varios países de la periferia y, por lo tanto, conserva plena vigencia. Pero además es central porque por su propia naturaleza es transversal a todo el aparato estatal y por tanto atraviesa los procesos de 
formulación, implementación y evaluación de un amplio abanico de políticas públicas ejecutadas por los estados.

En este contexto de cambio de siglo, además, el BM presenta variaciones en su orientación e intervención. Mendes Pereira (2017) identifica tres grandes novedades luego del 11 de septiembre de 2001. La primera es que pasó a priorizar las condicionalidades ex-ante en operaciones de única parte, según las cuales los recursos son liberados después de que los gobiernos clientes implementen los cambios acordados. Esto con la intención de mejorar la observación y el control de su asistencia financiera. En segundo lugar, a diferencia de los instrumentos diferenciados para el "combate contra la pobreza" de la década de los noventa, aparecen los Documentos Estratégicos de Reducción de la Pobreza, aplicados, en principio, en países pobres altamente endeudados. Por medio de su gestión el Banco asumió la coordinación del conjunto de la cooperación multilateral para el desarrollo. La tercera novedad es la creación en el año 2006 de un sistema de evaluación del ambiente institucional de los estados clientes llamado Country Policy and Institutional Assessment (CPIA). Tal sistema establece un ranking de estados elegibles para préstamos de ayuda internacional de acuerdo con las cualidades de sus políticas, a partir de 16 criterios organizados en cuatro grupos (Mendes Pereira, 2017).

Para profundizar en la caracterización del BM veamos algunos aspectos relacionados con: su ubicación en el marco de la llamada Ayuda Oficial para el Desarrollo (ODA por sus siglas en inglés: Oficial Development Aid), para identificar su peso relativo dentro de la ayuda internacional; la evolución de sus compromisos, para observar la trayectoria de su política de financiamiento; y la distribución sectorial de esos compromisos, para observar el peso relativo que tienen los diferentes sectores en esa misma política de financiamiento.

Comencemos por ubicar la asistencia financiera del BM en relación con algunas variables vinculadas a la ODA. Las contribuciones básicas de los países miembros del CAD (Comité de Ayuda para el Desarrollo) hacia las agencias multilaterales equivalió a 41725000000 de dólares en el año 2017.7 De este total, el Banco Mundial absorbió el 19\% (9000 000000 de dólares), sólo detrás de la Unión Europea, que obtuvo el 34 \% de los recursos (14000 000000 de dólares). Además, el desembolso bruto total del Grupo Banco Mundial equivale en promedio al $24 \%$ de los desembolsos brutos de la Ayuda para el Desarrollo durante el período 2014-2017. ${ }^{8}$ Es decir, los desembolsos del Grupo Banco Mundial representan casi la cuarta parte de los desembolsos totales canalizados por la Ayuda Oficial para el Desarrollo alrededor del mundo. Por su parte, los desembolsos brutos del BIRF y la AIF (o sea, el BM en stricto sensu) equivalen en promedio al $17 \%$ de los desembolsos brutos de la Ayuda Oficial para el Desarrollo en el mismo período. Ello también habla de un fuerte peso del financiamiento del BM en comparación con el conjunto del financiamiento oficial a escala global, como se observa en el siguiente cuadro: 
Tabla No1 Desembolsos brutos de AOD, GBM, BIRF Y AIF

Período 2014-2017 En millones de dólares (Valores corrientes)

\begin{tabular}{|l|r|r|r|r|}
\hline & \multicolumn{1}{|c|}{2014} & \multicolumn{1}{c|}{2015} & \multicolumn{1}{c|}{2016} & \multicolumn{1}{c|}{2017} \\
\hline ODA & 194.823 & 170.908 & 192.906 & 197.518 \\
\hline GBM (a) & 44398 & 44582 & 49039 & 43853 \\
\hline BIRF+AIF & 32193 & 31917 & 35723 & 30579 \\
\hline GBM/ODA & $23 \%$ & $26 \%$ & $25 \%$ & $22 \%$ \\
\hline BIRF+AIF/ODA & $17 \%$ & $19 \%$ & $19 \%$ & $15 \%$ \\
\hline
\end{tabular}

Elaboración propia en base a datos de OCDE e Informe Anual 2018 del BM. Nota: a) Se incluyen los desembolsos del BIRF, la AIF, IFC y los fondos fiduciarios ejecutados por el receptor, El dato de 2017 de la AIF incluye el compromiso y desembolso de una donación de USD 50 millones para el mecanismo de Financiamiento de Emergencia para Casos de

Pandemia. La AOD incluye los desembolsos de los países miembros como no miembros del Comité de Ayuda para el Desarrollo, y también los desembolsos multilaterales (correspondiente, según los datos de la OCDE, únicamente a la UE).

Sigamos con la evolución de los compromisos del BM en las últimas tres décadas, tomando como puntos de referencia los años 1991, 1998, 2008 y 2018. Los compromisos del BIRF para el año 1991 ascienden a los 16000000000 de dólares, aumentan hacia el año 1998 llegando a 21000000000 de dólares, para hacia el año 2008 disminuir a 13000000000 y luego volver a ascender hasta los 23000000000 hacia el año 2018. Los compromisos de la AIF experimentan una evolución gradual de aumento sostenido a lo largo del período de la serie, que se inicia con 6000000000 de dólares en 1991 y culminando con 24000000000 de dólares en 2018. De conjunto, los compromisos del BIRF y la AIF comienzan la serie equivaliendo a 22000000000 de dólares en 1991, luego ascienden a 28000 000000 en 1998, descienden a 24703000000 hacia el año 2008 y culminan con 47000000000 hacia el año 2018. A pesar de que la serie no permite identificar las ondas intermedias de la evolución entre cada uno de los años de referencia, sí aporta a reconocer tendencias generales del período. En términos generales, podemos identificar, si tomamos la suma de los montos de compromisos del BIRF y la AIF, una trayectoria en la que los compromisos aumentan durante la década de los años noventa de auge del endeudamiento de los países capitalistas atrasados y de transición capitalista en países exsoviéticos; disminuyen en la primera década del nuevo siglo luego de varias crisis nacionales y de que en paralelo el alza de los commodities relaje el frente externo en varios países atrasados; cobra nuevo impulso tras el estallido global de la crisis en 2007-2008 que agudiza conflictos políticos y geo-políticos en varias partes del mundo (por ejemplo, África), y que inicia un nuevo ciclo de expansión y socialización de las deudas (Cantamutto y Castiglione, 2021). Esta tendencia general se aprecia en el Gráfico №1. 
Gráfico N¹ Montos de los compromisos del BIRF y AIF En millones de dólares (valores corrientes)

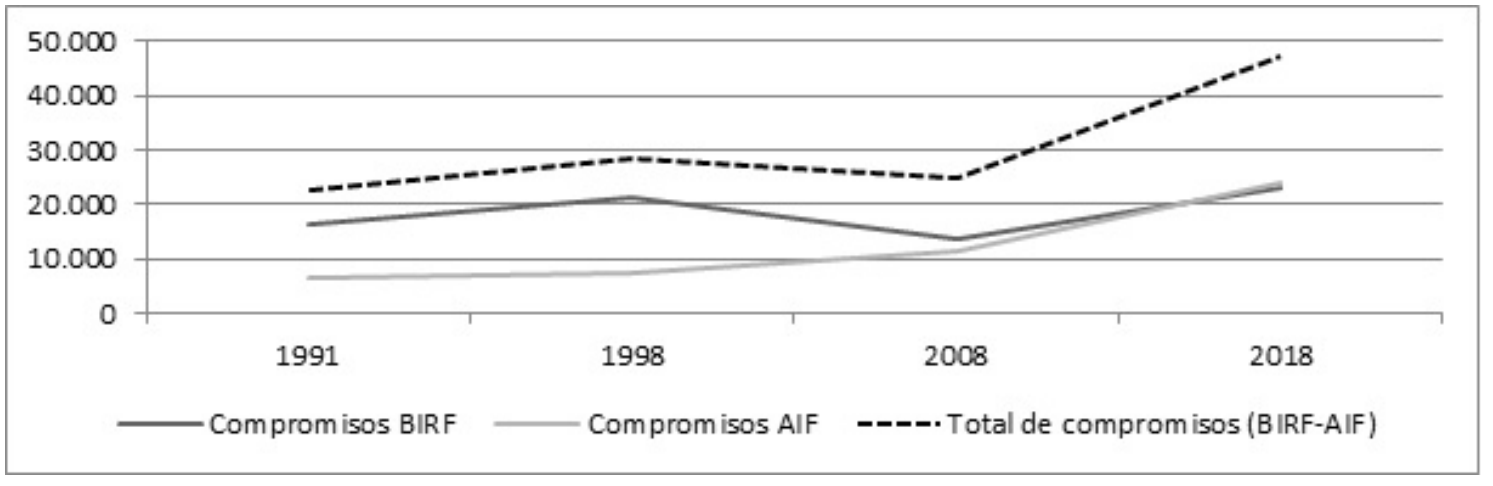

Fuente: Elaboración propia en base a Informes Anuales del Banco Mundial (1991, 1998,

2010, 2018). Los datos corresponden a los ejercicios anuales de cada año con cierre en junio.

Veamos, por último, la distribución sectorial de los compromisos del Banco Mundial. Al cambiar con frecuencia la metodología de clasificación del propio organismo, no existe una taxonomía única de sectores para un período extenso. Por esta razón, tomamos los últimos cuatro ejercicios anuales para graficar esta dimensión de la orientación e intervención del Banco actual. Si tomamos los promedios de la serie (2014-2018), la primera tendencia general que observamos es la mayor gravitación de los sectores "energía e industrias extractivas", "administración pública”, "transporte” y "abastecimiento de agua, saneamiento y gestión de desechos". Cada uno de los dos primeros sectores (energía e industria extractivas y administración pública) sostiene una participación del $15 \%$ promedio a lo largo de la serie. Esto significa, en términos absolutos, compromisos por montos de alrededor de 7000000000 de dólares para el año 2018. El sector "transporte" sostiene una participación promedio del $13 \%$ a lo largo del período, aunque en el último año su participación en la cartera baja con fuerza (8\%). Por último, el sector de abastecimiento de agua y saneamiento sostiene una participación del $11 \%$ promedio a lo largo del período. Por separado el peso de los sectores de salud, educación y protección social (asistencia social) durante el mismo período es escaso: su participación en la cartera es de $6 \%, 8$ $\%$ y $8 \%$, respectivamente. Pero en conjunto los tres sectores correspondientes al área de la política social (educación, salud y protección social) suman el $22 \%$ promedio de participación en la cartera de compromisos del BM a lo largo del período. En términos absolutos, el monto de compromisos de los tres sectores juntos equivale a 12990000000 de dólares para el año 2018. 
Gráfico No2 Compromisos del BIRF y AIF. Ejercicios 2014-2018 En millones de dólares (valores corrientes)

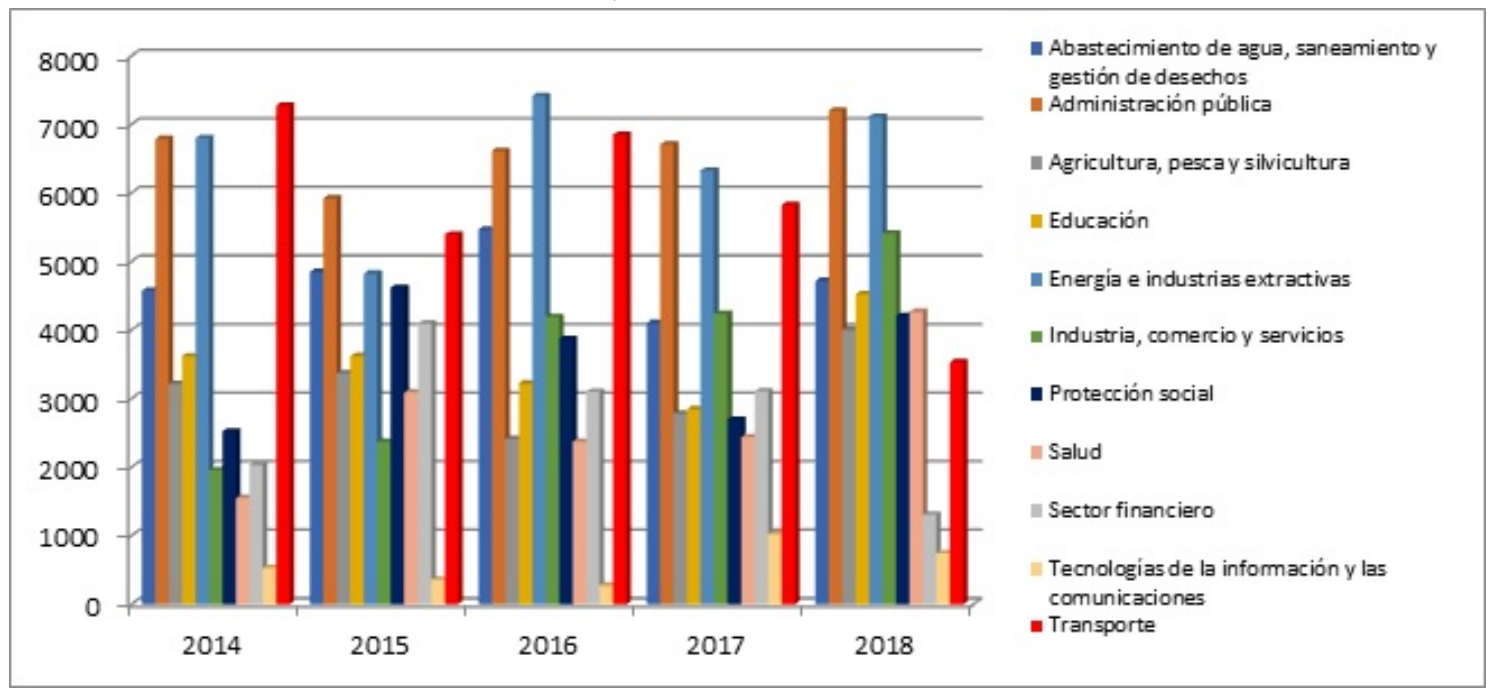

Fuente: elaboración propia en base a Informe Anual 2018 del BM. Nota: En el ejercicio 2017 el BM cambio la taxonomía utilizada en ejercicios anteriores, por lo que el Banco realizó una reclasificación de los sectores tal como aparecían en los ejercicios previos a 2017.

\section{3 - La política del Banco Mundial hacia las políticas sociales y la cuestión social}

Tal como esbozamos más arriba, en la década del sesenta el Banco incorpora a su orientación político-financiera la intervención sobre la cuestión social y el área de las políticas sociales. Aunque el proceso comenzó a inicios de la década, se fortalece hacia fines de ésta con la asunción de Robert McNamara en la presidencia del Banco, en abril de 1968. Durante los años 1973-1974 la "pobreza rural absoluta" se definió como el principal objeto de la intervención del Banco a través de los nuevos proyectos de "desarrollo rural integrado" (DRI). El "combate a la pobreza" como leitmotiv del Banco nació en este momento.' El énfasis de la orientación hacia la reducción de la pobreza, y sobre todo hacia la pobreza rural, se explica, según Mendes Pereira (2012), por tres factores. En primer lugar, la lectura de la centralidad del campesinado en las dinámicas políticas de los países atrasados por parte de la política exterior norteamericana. Por su gravitación en la estabilidad política, el campesinado y el área rural se convertían en puntos estratégicos de intervención. En segundo lugar, la aparición de un principio de cuestionamiento a la concepción que hasta ese entonces predominaba en el Banco: la visión de la reducción de la pobreza vía el "efecto de goteo hacia abajo". Es decir, la idea de que, logrado el inicio del proceso de desarrollo capitalista en los países atrasados -el "despegue" vía industrialización-, sobrevendría la reducción de la pobreza. En tercer lugar, el establecimiento en el Congreso estadounidense de la iniciativa New Directions en 1973. La nueva directiva, que planteaba reorientar la ayuda exterior bilateral, procuraba resolver el problema de la reducción de la pobreza sobre la base de créditos a pequeños agricultores. Esto, claro, estaba en sintonía con otras estrategias (por ejemplo, la Alianza para el Progreso de 1971). El énfasis en el sector rural y la pobreza en estas áreas le permitía al Banco localizar el problema y promover iniciativas como la reforma agraria, el crédito para los pequeños propietarios, la educación rural, los proyectos de irrigación y servicios de salud como medios específicos de reducir 
gradualmente la pobreza. En este marco, el Banco no sólo instala el problema de la pobreza, sino también una concepción de ella (la de que se reduciría aumentando la productividad de los pobres) y una forma de estimarla: la pobreza absoluta rural y urbana como unidad de medida (Mendes Pereira, 2010; Mendes Pereira, 2012). Pero la orientación no era sólo hacia el espacio rural sino también hacia el área urbana. La fundamentación para este último aspecto se encuentra con claridad en este pasaje de McNamara:

\begin{abstract}
"Históricamente, la violencia y los levantamientos civiles son más comunes en las ciudades que en el campo. Las frustraciones que supuran entre los pobres urbanos son rápidamente explotadas por los extremistas políticos. Si las ciudades no comienzan a tratar de manera más constructiva con la pobreza, la pobreza bien podría comenzar a tratar más destructivamente con las ciudades. No es un problema que se incline al aplazamiento político” (McNamara, 1981 [1975], p. 316 citado por Mendes Pereira, 2012, p. 124).
\end{abstract}

Con esto señalamos que la orientación política del Banco Mundial hacia la cuestión social en sus países miembros prestatarios hunde sus raíces en los sesenta y la primera década de los setenta, en correlación con el trazo de la historia dominado por el alza de la lucha de clases a lo largo del mundo y la crisis del capitalismo de posguerra. La bandera de la lucha contra la pobreza a partir de este momento no sólo seguirá flameando hasta el día de hoy, sino que cobrará creciente centralidad en la intervención del BM. ${ }^{10}$ Pero vayamos más específicamente a la orientación política hacia la cuestión social que construye el Banco Mundial en el período que se abre con la ofensiva neoconservadora a escala global.

La orientación política del Banco Mundial hacia la cuestión social durante la primera etapa de la década de los noventa se inscribe en la orientación general signada por el Consenso de Washington. El Informe Anual de 1991, en línea con el Informe para el Desarrollo Mundial, continúa colocando a la reducción de la pobreza en la máxima jerarquía de la intervención del BM. Para este objetivo propugna una estrategia basada en dos planos. El primero consiste en "fomentar un crecimiento económico de amplia base por medio del uso productivo del bien que los pobres poseen en mayor abundancia, a saber, su trabajo" (BM, 1991, p.11). El segundo plano exige "inversiones en servicios sociales -en particular, servicios básicos de educación y salud, planificación familiar y nutrición-, con objeto de mejorar las condiciones de vida de la población pobre y aumentar su capacidad para aprovechar las oportunidades de obtención de ingresos que surjan del crecimiento económico" (BM, 1991, p. 11). O sea, enfatizar la acumulación de capital humano de los pobres como base para que aprovechen el crecimiento económico mediante el trabajo. En razón de que bajo estos dos planos de la estrategia queda excluido un amplio sujeto (las personas adultas mayores, los enfermos, las viudas, etc.), el BM plantea como aspecto complementario la construcción de un "sistema de transferencias y redes de protección social". Es decir, sistemas de ayuda que permitan, según el Banco, "salir adelante en situaciones de dificultades y calamidades temporales".

Por supuesto, en esta orientación política para que el crecimiento económico se desate primero se debían desanudar las rigideces a las que estaba sometido el mercado. De esta forma, el crecimiento económico y su derivación en aumento del empleo -la llamada "utilización del activo trabajo de los pobres"- 
se daría luego de liberalizar el mercado en general y el mercado de trabajo en particular. Para el Banco las intervenciones gubernamentales típicas obstaculizan la inversión y, por consiguiente, punzan a la baja la demanda de empleo. Pero además de recomendar la eliminación de las regulaciones distorsivas del mercado, sugiere incorporar a los propios beneficiarios en el diseño de los programas sociales. ${ }^{11}$

Al mismo tiempo critica la escasa focalización del gasto público en los servicios sociales. En esta línea, argumenta que en la mayoría de los países el gasto es desviado a la gente que no lo necesita. Y, en consonancia, recomienda que el gasto público en servicios sociales se concentre en los sectores pobres. Además, el gasto en salud debe ser el gasto en salud básica (preventiva o primaria) y el gasto en educación no debe ser en el nivel superior, sino que se debe priorizar el gasto en la educación primaria, ya que beneficiarían más a los pobres.

Hacia el cambio de siglo, el Banco reajusta su orientación política a la luz de los resultados que venía arrojando la implementación de las reformas neoliberales en varios países bajo su asistencia técnica-financiera. En el Informe para el Desarrollo Mundial 2000/2001 da cuenta de esta revisión. En efecto, plantea que los dos pilares de su estrategia hacia la pobreza diseñada a principios de los noventa (uso intensivo de la mano de obra y amplia provisión de servicios sociales, a los que se agregaba redes de protección social) deben ser complementados. En este sentido, agrega tres nuevos ejes en la estrategia de reducción de la pobreza: "oportunidad", "empoderamiento" y "seguridad". Como plantea el informe 2000/01:

“La experiencia que inspiró el Informe de 1990, desde la perspectiva de 1989, era la experiencia contradictoria de los años setenta y ochenta en Asia oriental, donde la pobreza había retrocedido fuertemente, y de África, América Latina y Asia meridional, donde había disminuido menos o incluso aumentado. ¿Por qué Indonesia había conseguido mejores resultados que Brasil en los años setenta y ochenta en su intento de reducir la pobreza en sus distintas dimensiones - el ingreso, pero también la educación y la salud? La respuesta era un crecimiento basado en la mano de obra y una amplia provisión de servicios sociales - la doble estrategia del Informe." (Banco Mundial, 2001, p. 32).

Para el Banco, la experiencia de los años noventa confirma la importancia del crecimiento y la acumulación de capital humano en los pobres para la reducción de la pobreza, pero además agrega nuevos elementos a la ecuación. El primero es que el crecimiento no va acompañado necesariamente de reducción de la pobreza. $\mathrm{Al}$ menos, por dos cuestiones. Porque no está acompañado de instituciones eficientes (cita el caso de las reformas en los países de la ex Unión Soviética), o bien, porque la pauta de crecimiento se apoya en conocimiento especializado, que no incorpora mano de obra no calificada. Otro elemento marcado son los límites de la provisión de los servicios sociales. De nuevo estos límites aparecen atados a las capacidades institucionales locales, como también a la estructura del mercado, o a las características de la influencia política. Además, reconoce que los flujos de capital privado refuerzan "tendencias económicas positivas", castigando países donde la situación económica no es favorable. Con el objetivo de superar el anterior marco de acción, propone un nuevo marco de actuación basado en tres áreas: 
“Oportunidad: Hacer llegar las oportunidades económicas a los pobres estimulando el crecimiento general y multiplicando sus activos y haciéndolos más rentables, mediante una combinación de intervenciones en el mercado y en otras esferas

Empoderamiento: Conseguir instituciones estatales más dispuestas a rendir cuentas y a tener en consideración las necesidades de los pobres, fortalecer la participación de éstos en los procesos políticos y en las decisiones locales, y eliminar los obstáculos sociales que se derivan de las diferencias por razón de sexo, etnia, raza y condición social

Seguridad: Reducir la vulnerabilidad de los pobres a la mala salud, las crisis económicas, las perturbaciones provocadas por las intervenciones estatales, los desastres naturales y la violencia, y ayudarles a superar las crisis cuando se producen" (BM, 2001, p.33).

Con oportunidad hace referencia a ayudar a los pobres a aumentar sus activos (desde educación y salud, hasta servicios financieros y redes interpersonales). Con empoderamiento se refiere a aumentar la capacidad de los pobres de influir en las instituciones estatales que inciden en su vida, reforzando su participación en los procesos políticos y las decisiones locales. También significa aumentar los activos para que puedan intervenir en los mercados. En este punto, se incluye la capacidad administrativa y reductora de la corrupción. Con esto hace referencia a racionalizar la estructura del sector público (mejorar los sistemas de gestión en un sentido de eficiencia y transparencia), y a que la sociedad civil participe en la planificación, supervisión, evaluación de los programas y las políticas de las autoridades públicas. En este marco, también entra el impulso a la descentralización: "La descentralización puede conseguir que las instituciones públicas respondan mejor a las necesidades de los pobres, pero sólo si se permite que éstos exijan responsabilidades a los funcionarios públicos y si se garantiza su participación en el proceso de desarrollo" (BM, 2001, p. 206). ${ }^{12}$ La tercera área planteada, la seguridad, se traduce en el respaldo a las instituciones de "gestión de riesgos" de los pobres. Esto es, instrumentos como el seguro médico, la asistencia y las pensiones para los ancianos, el seguro de desempleo, los programas de asistencia social vinculados al trabajo, los fondos sociales, los programas de microfinanciamiento y las transferencias de recursos en efectivo. Se plantea un "sistema modular" de control de los riesgos que adapta las medidas de protección social a la situación específica de cada país.

En términos generales, continúa planteando una correlación entre reformas (medidas en volumen del comercio, tasas arancelarias, tasas de inflación y déficits presupuestarios) y tasa de crecimiento económico. Donde esta correlación no se da se explica por falta de instituciones que lo respalden, por errores en la secuencia de los cambios, o por grupos dominantes que monopolizan las reformas (esto último, en referencia a los países exsoviéticos). Advierte sobre los "costos sociales" que pueden implicar las reformas en los países, y plantea proteger los activos de los pobres y el capital humano de sus hijos. Por otro lado, argumenta que las posibilidades de reforma como medio de facilitar el acceso de los pobres a los mercados puede darse en tres esferas: "el alivio de la carga de reglamentaciones" (baja de costos administrativos, sistema tributario), la promoción de normas laborales básicas (flexibilidad laboral) y la ampliación de servicios de financiamiento (búsqueda de mecanismos para facilitar el crédito a los pobres, tanto para costos familiares como para microempresas). Otro aspecto central del diagnóstico es que los pobres cuentan con baja capacidad 
para financiar la educación, la salud y otras inversiones relacionadas con otros activos. En este marco, plantea que el estado debe contribuir a ampliar los activos de los pobres, porque los mercados no funcionan en favor de ellos, debido a su aislamiento físico como a las insuficiencias del mercado en los sectores financieros, de salud, entre otros. La política pública puede reducir las desigualdades iniciales y aumentar las oportunidades para que los pobres se beneficien del crecimiento. Continúa, a su vez, sosteniendo la necesidad de focalizar el gasto público en los pobres, como también la necesidad de incorporar su participación en los programas sociales. ${ }^{13}$ Este último punto es jerarquizado como forma de promover la acumulación de activos. La participación "debe fomentarse con iniciativas que den mayor resonancia a la opinión de los grupos pobres empeñados en combatir la estratificación o el estigma social. Ello significa que la participación debe plantearse en un contexto institucional más amplio" (BM, 2001, p. 88). ${ }^{14}$ Otro aspecto en este punto es que el estado debe construir coaliciones a favor de la pobreza, es decir, convencer a la comunidad de la coincidencia de intereses entre los pobres y el resto de la población.

Lo que podemos identificar, en base a los documentos de principios del nuevo milenio, es una continuidad de lo central de la orientación política tejida durante los años noventa por el mismo BM. En efecto, permanecen las recomendaciones básicas de su credo hacia la cuestión social y el área de las políticas sociales: focalización en los sectores pobres, énfasis en la acumulación de capital humano para la "salida de la pobreza", descentralización de los servicios sociales y participación de la sociedad civil en los programas sociales. Con independencia de la renovación semántica, se le aggiorna el nuevo énfasis en la calidad institucional, la eficiencia y la transparencia del estado sustentado en el neo-institucionalismo. Además, se le adosa una nueva preocupación por la legitimidad portada por los programas sociales en la sociedad, para lo que llama a construir coaliciones en favor de estos.

Por otro lado, como lo introdujimos en el anterior apartado, en términos de la intervención del BM encontramos los llamados Poverty Reduction Strategy Papers (PRSP), lanzados en septiembre de 1999. Presentados como un nuevo enfoque por parte del BM y el FMI, estos documentos reemplazan los Policy Framework Papers elaborados por el BM, el FMI y los gobiernos del país prestatario. Ruckert (2010) plantea que el enfoque plasmado en estos documentos combina la continuidad del apoyo a la privatización de los servicios sociales con un nuevo énfasis sobre la dirección de los subsidios a las personas pobres. Los PRSP fueron originalmente planteados como condición de la iniciativa Países Pobres Altamente Endeudados, una estrategia del FMI y el BM de 1996. Como señalan Malaluan y Guttal (2003), el rol de los PRSP va más allá de los préstamos e incluye a las donaciones. Se convirtieron en instrumentos clave mediante los cuales la mayor parte de los donantes del mundo se relacionaba con los países más pobres receptores de ayuda. Sin la aprobación de un PRSP por parte de las juntas ejecutivas del BM y del FMI, un país podía ser virtualmente abandonado en la ayuda internacional, el comercio y las finanzas. Los últimos PRSP acordados datan del año 2010, en el marco de los efectos de la crisis mundial, y fueron planteados para cuatro países africanos: República del Congo, Kenia, Togo y Uganda. ${ }^{15}$ 
También en el contexto de la crisis mundial del año 2008 el Banco Mundial estableció el Programa para Hacer Frente a la Crisis Mundial de los Alimentos (GFRP, por sus siglas en inglés). La iniciativa duró hasta mitad del año 2012 y consistió en financiamiento por vía expedita del BIRF como de la AIF y en donaciones de fondos fiduciarios para apoyar distintas políticas de alimentos. Específicamente, el GFRP financió: i) políticas de precios de los alimentos y estabilización del mercado; ii) medidas de protección social para garantizar el acceso a los alimentos y reducir al mínimo el impacto nutricional de la crisis en los pobres y vulnerables; iii) mejora de la respuesta en materia de producción y comercialización de alimentos a nivel nacional. En conjunto, los recursos alcanzaron a alrededor de 49 países y, según el BM, beneficiaron a 65,9 millones de personas. ${ }^{16}$

Por otra parte, el Banco Mundial será la principal organización internacional en la articulación de la internacionalización del paradigma de los Programas de Transferencias Monetarias Condicionadas (PTMC) durante las últimas dos décadas. Como mostramos en otro trabajo (Fernández, 2021a), su capacidad de organización y difusión del paradigma de los PTMC se asienta en su amplio radio de intervención a escala global y en el conjunto de mecanismos desde los cuales opera: la asistencia técnico-financiera ${ }^{17}$ y las plataformas de divulgación y aprendizaje (desde la organización de foros hasta la generación de insumos teóricos plasmados en documentos de evaluación). ${ }^{18}$ Desde esta potencialidad, logró estandarizar el paradigma bajo premisas como la centralización del instrumental de la asistencia, el reforzamiento de los mecanismos que garantizan el cumplimiento de las condicionalidades, la transparencia en la asignación de las prestaciones, el fortalecimiento de los sistemas de evaluación y monitoreo, y el impulso de mecanismos de egreso, entre otros aspectos (Fernández, 2021a). Además del neoinstitucionalismo como marco teórico que guía las transformaciones político-institucionales necesarias para ganar eficiencia y eficacia en la instrumentación de estos programas, en el mismo proceso también se consolida la teoría del capital humano como marco teórico que fundamenta las condicionalidades de aquellos programas. De esta forma, la teoría neoclásica, y en particular la teoría del capital humano acuñada por Becker, no sólo organiza la concepción general del BM hacia las políticas sociales y la cuestión social, sino que también es el principal fundamento teórico-político de las condicionalidades -sobre la teoría Cfr. Becker y Tomes (1994) y Lindahl, Palme, Sandgren-Massih y Sjögren (2014)-.

El énfasis en la focalización de las políticas sociales en las personas pobres no debe entenderse como un planteo de estrechez en la cobertura social de los programas sociales. Por el contrario, en las últimas dos décadas, madura en el BM una orientación política que hace hincapié en la necesidad de expandir programas de asistencia social masiva a lo largo del mundo. Aunque puede ser registrada antes, esta orientación queda bien plasmada en la Alianza Mundial para la protección social universal, declarada en septiembre de 2016 en la Asamblea General de la ONU y acordada entre otros organismos por la OIT (Organización Internacional del Trabajo) y el propio BM. En este contexto, el expresidente del Grupo Banco Mundial, Jim Yong Kim, declaraba: ““(...) En la actualidad, si bien numerosos países en desarrollo ya han instaurado regímenes universales concebidos para garantizar que nadie sea dejado atrás, 
sólo una de cada cinco personas pobres en los países de más bajos ingresos está cubierta por alguna forma de protección. Es profundamente significativo que nos hayamos reunido para ayudar colectivamente a los países a colmar estos déficits de cobertura." (septiembre de 2016). ${ }^{19}$

En su último informe anual de 2018 el Banco ratifica sus dos objetivos centrales: poner fin a la pobreza extrema antes de fines de 2030 e impulsar la prosperidad compartida del $40 \%$ más pobre de la población del mundo. Estos objetivos, y principalmente el primero, se inscriben en la "Agenda 2030 de Desarrollo Sostenible", acordada en septiembre de 2015 en la Cumbre de Desarrollo Sostenido en el marco de la ONU. ${ }^{20}$ La estrategia para lograr estos objetivos se apuntala en acelerar el crecimiento económico inclusivo y sostenible, generar resiliencia ante las crisis y las amenazas, y ayudar a los países clientes a invertir en las personas que habitan en ellos. ${ }^{21}$

También de modo reciente corroboramos que la orientación política del Banco hacia el campo de la política social continúa amarrada a la apuesta por los activos de las personas pobres. Esto se refleja en la iniciativa de elaboración del Índice de Capital Humano, que califica a los países de acuerdo con lo adecuado de sus inversiones en el capital humano de la próxima generación. La iniciativa se denomina Proyecto sobre Capital Humano (PCH) y descansa en el diagnóstico de una "brecha de capital humano" crítica:

"Cientos de millones de niños y jóvenes no aprenden lo suficiente como para prepararse para los empleos del futuro, los sistemas de salud deficientes no proporcionan cobertura universal, y cuatro de cada cinco personas pobres en los países de ingreso bajo aún no tienen acceso a las redes de protección social. Por otro lado, la tecnología está cambiando la naturaleza misma del trabajo, por lo que se vuelve aún más urgente realizar inversiones de calidad en las personas y en sus capacidades" (BM, 2018, p. 22).

El Índice de Capital Humano que sostiene la iniciativa se dio a conocer a fines de 2018. El objetivo es que mida la supervivencia (¿los niños que nacen hoy sobrevivirán hasta la edad escolar?), la escolaridad (¿qué niveles educativos alcanzarán los niños y cuánto aprenderán?) y la salud (cuando dejen la escuela, ¿tendrán buena salud y estarán listos para continuar con sus estudios o para trabajar?). Esta centralidad otorgada a la formación de capital humano por el Banco Mundial explica la prioridad de su inversión en los primeros años de vida. ${ }^{22}$ O sea, la centralidad otorgada a la inversión en el estadio de formación de la mercancía fuerza de trabajo. En otro informe de la organización ${ }^{23}$, destinado a la región de América Latina y el Caribe, se detalla en este sentido:

\footnotetext{
"Cuando los programas sociales finalmente llegan a la población adulta, algunas personas crónicamente pobres pueden ya no disponer de las destrezas y la actitud mental requeridas para escapar de la pobreza de un modo sostenible. Para las políticas públicas esto representa tanto un desafío como una oportunidad para romper decididamente con el ciclo de la pobreza crónica en una etapa temprana del ciclo de vida. Por eso el diálogo sobre politicas emergentes y la integración del desarrollo de la primera infancia en la agenda de desarrollo social de la región son bienvenidos, y deberían ser aún más propagados" (Vakis, Rigolini y Lucchetti, 2015, p. 32; cursivas nuestras).
}

En este mismo informe también se señala la centralidad de los programas de empleo en la reducción de la pobreza; en concreto, los programas de capacitación 
laboral y reinserción laboral. Es decir, que actúan sobre la oferta en el mercado laboral. De forma complementaria también se acentúa una línea de intervención psico-social sobre los pobres:

"En el diseño de las políticas públicas es necesario incluir estrategias para revertir las aspiraciones deprimidas de los pobres crónicos y tomar en cuenta su estado mental. $\mathrm{Si}$ el estado mental de los pobres crónicos no es atendido, estos se escurrirán por los resquicios del sistema de redes de seguridad y se abstendrán de inscribirse en los programas sociales, o de cumplir con sus corresponsabilidades, con lo cual terminarán en última instancia siendo sancionados. Un programa social puede ayudar a los pobres a superar su estado mental y sus bajas aspiraciones para que se enganchen con el proceso" (Vakis, Rigolini y Lucchetti, 2015, p. 34; cursivas nuestras).

Por otro lado, el Banco plantea:

"Es necesario, por lo tanto, ir más allá del enfoque clásico de asistencia social, de corte "pasivo", donde la cobertura está dictada por consideraciones presupuestales, el nivel de pobreza y el supuesto de que los pobres buscarán y se inscribirán proactiva y exitosamente en los programas sociales, y orientarse hacia enfoques más "activos", que busquen a los pobres extremos y crónicos, los ayuden a aprovechar el sistema de protección social de manera eficiente y los asistan con la identificación de sus propios objetivos de desarrollo" (Vakis, Rigolini y Lucchetti, 2015, p. 37).

Y al mismo tiempo:

"Este enfoque merece cierta atención pues representa un giro: del paradigma tradicional de la asistencia social, que consiste en brindar a los pobres una amplia gama de bienes y servicios, hacia un enfoque más personalizado, que aspira a suministrar a las personas las herramientas que necesitan para enfrentar sus retos especificos. A diferencia del enfoque clásico de protección social, según el cual las familias crónicamente pobres tienen que postular a los beneficios, los servicios de intermediación social colocan a las familias en el centro, al identificar y abordar activamente a los pobres crónicos, y garantizarles un acceso prioritario a programas tanto existentes como nuevos". (Vakis, Rigolini y Lucchetti, 2015, p. 38; cursivas nuestras).

Dentro de este paraguas, programas de transferencias monetarias condicionadas, como el Chile Solidario o Juntos de Colombia, son colocados como ejemplos en la utilización de los servicios de intermediación.

De este modo, identificamos una orientación política que lejos de abjurar de su tradición, la rejuvenece con nuevas palabras e instrumentos. Debajo del tratamiento de belleza, claro, continúa una vieja matriz liberal-conservadora de prescripción política que sostiene la individualización y psicologización de los problemas sociales, y el disciplinamiento de la clase trabajadora pauperizada vía la tutela, el control y la modelación de sus conductas. En este sentido, la orientación hacia el área de la política social en general y hacia la asistencia social en particular combina la selectividad estratégica en la formación de la mercancía fuerza de trabajo con el impulso de mecanismos de subordinación política de la clase trabajadora vía la incorporación de sus intereses más inmediatos. Por un lado, se trata del sostenimiento de atributos productivos mínimos (educación básica para una calificación mínima y salud básica para una mínima disposición físicobiológica del cuerpo que porta la mercancía fuerza de trabajo) de la población pobre en combinación con asistencia monetaria que apuntale sus prácticas de supervivencia en tanto sobrantes para las necesidades medias del capital (Marx, 2009). Por otro lado, se trata de contenerla políticamente incorporando sus intereses más inmediatos, en tanto población potencialmente conflictiva que 
puede entorpecer las condiciones de dominación política necesarias para la acumulación de capital en los espacios nacionales de valor.

Por último, a nivel de la asistencia técnico-financiera observamos la consolidación, en las últimas dos décadas, de la política del BM hacia las políticas sociales. Para el año 1991, los compromisos del BIRF y de la AIF hacia el área de política social representan el $17 \%$ del total, para el año 1998 representan el $23 \%$, mientras que para el año 2008 se desplazan al $14 \%$, y para 2018 ascienden al 28 $\% .{ }^{24}$ En este sentido, la política técnico-financiera hacia las políticas sociales del $\mathrm{BM}$ se correlaciona con la centralidad que ocupa en su orientación plasmada en documentos y declaraciones. Veamos el siguiente gráfico:

Gráfico No3 Relación Compromisos BIRF y AIF en el área de política social/

Total de compromisos BIRF-AIF Período 1991-2018 Valores en porcentajes

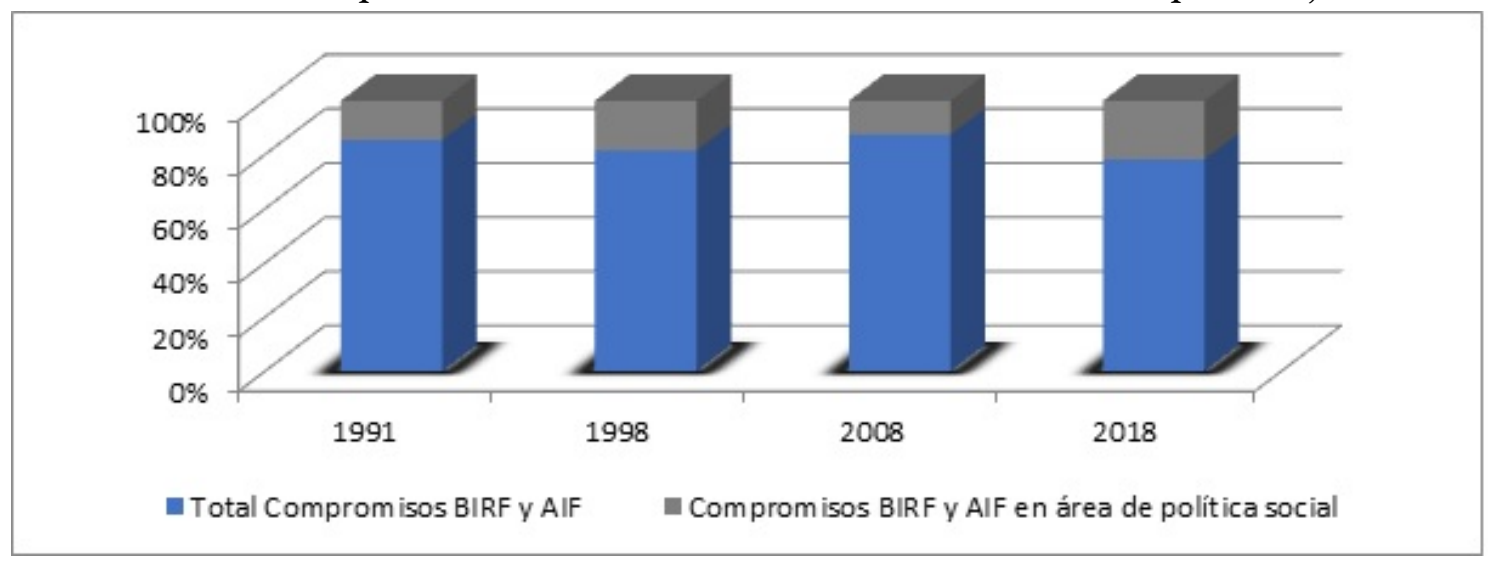

Elaboración propia en base a Informes Anuales. Nota: para el año 1991 se incluye en área social lo que el BM clasifica en Educación, Población, Salud y Nutrición. Para el año 1998 se incluye: Agricultura, Educación, Población, Salud y Nutrición, Sector Social. Para 2008 se incluye: Salud y Servicios Sociales, Educación. Para el año 2018 se incluye: Salud y Servicios Sociales, Sector social, y Educación.

\section{4-Conclusiones}

En el trabajo planteamos que el comienzo de la política del Banco Mundial hacia las políticas sociales se da a inicios de la década del sesenta en un marco de alza de la lucha de clases y de radicalización política en todo el mundo. También señalamos la manera en que dicha política se profundiza durante la etapa de ofensiva del capital contra el trabajo, el neoliberalismo, que condujo la salida de la crisis del capitalismo de posguerra. El BM fue protagonista estrella en la articulación internacional de aquella ofensiva que, basada en la disciplina monetaria, reestructuró el capitalismo a nivel mundial sobre la base de aumentar la competencia en el mercado: entre capitalistas y, al mismo tiempo, entre trabajadores (Piva, 2020). En este marco, planteó en los años noventa una orientación hacia la política social que consistió básicamente en promover la privatización de servicios sociales como la educación y la salud, la focalización de estos servicios en su carácter público en las personas pobres y la construcción de "redes de protección social" basadas en programas asistenciales para aquella porción de la población que se mantuviera repelida del mercado laboral. Destacamos su énfasis en fortalecer el capital humano de la población pobre para romper el llamado ciclo intergeneracional de la pobreza, que se sostiene en la matriz neoclásica de la teoría del capital humano. Bajo esta 
orientación hacia las políticas sociales, modernizó los viejos lineamientos hacia ellas, sustentados en una perspectiva de individualización de los problemas sociales y de búsqueda de ajuste bio-psico-social de la población pobre a las condiciones de explotación y dominación vigentes. Se trató, ni más ni menos, de la actualización de viejos mecanismos de subalternización de la capa pobre de la clase trabajadora.

En segunda instancia, mostramos cómo dicha orientación continúa y se consolida en las últimas dos décadas del nuevo milenio. Hicimos énfasis en que la importancia dada a la política asistencial por parte del organismo -las llamadas "redes de protección social", expresadas ejemplarmente en los PTMCresponde a dos requerimientos centrales de la acumulación y la dominación capitalista contemporánea. Por un lado, la necesidad de sostener educación y salud básicas para conservar atributos productivos mínimos de la población pobre que recibe dicha asistencia. Esta población en tanto sobrante para las necesidades productivas medias del capital continúa siendo requerida por el mercado, en su modalidad estancada, fluctuante o latente (Marx, 2009). Por otro lado, la masa de población pobre es vista como potencialmente conflictiva, por lo que su contención política vía concesiones materiales mínimas continúa siendo una necesidad de primer orden de la dominación política. De este modo, estos dos requerimientos de la acumulación y la dominación capitalista, mediados por la lucha de clases, están en el centro de la política del Banco Mundial hacia las políticas sociales en general y hacia las políticas asistenciales en particular. Son requerimientos que están presentes desde hace décadas, pero que desde los años noventa reciben una respuesta política más específica, consolidada en los últimos veinte años.

También señalamos que, en la actualidad, la orientación de focalización de la asistencia social hacia los pobres no quiere decir asistencia mínima, sino asistencia masiva. Este impulso a la asistencia social masiva por parte del BM y otras organizaciones internacionales revela la centralidad que adquiere a escala global la respuesta a aquellos requerimientos de la reproducción capitalista.

Por último, señalemos que, si bien el BM articula una política global, posee una gran versatilidad para adaptarse a las dinámicas políticas y económicas que surcan el proceso histórico de los distintos países prestatarios. Por ejemplo, en nuestro país demostró capacidad de adaptación a la coyuntura política y económica abierta tras la crisis de acumulación y dominación de principios de siglo, acompañando el proceso de recomposición del orden político que siguió a dicha crisis. ${ }^{25}$

\section{Fuentes}

Overseas Development Institute (ODI) (2018) A guide to multilateral development Banks, Lars Engen and Annalisa Prizzon, London.

World Bank. Informe Anual 1991

World Bank. Informe Anual 1998

World Bank. Informe Anual 2000/2001

World Bank. Informe Anual 2010

World Bank. Informe Anual 2018 
World Bank. ON THE EDGE OF UNCERTAINTY Poverty Reduction in Latin America and the Caribbean during the Great Recession and Beyond Public. WB- 2011.

World Bank (1998). Más allá del consenso de Washington: la hora de la reforma institucional. Washington.

Oxford Poverty and Human Development Initiative (2018). Global Multidimensional Poverty Index 2018: The Most Detailed Picture To Date of the World's Poorest People, University of Oxford, UK. (consultado 1/5/2019). https://www.imf.org/external/np/jsa/index.aspx\#U (consultado 10/2020) https://www.bancomundial.org/es/results/2013/04/11/global-food-crisis-r esponse---program-results-profile (consultado 10/2020).

http://www.bancomundial.org/es/publication/human-capital (consultado 10/2020)

http://www.realidadayuda.org/ (consultado 1/5/2019).

https://www.ilo.org/global/about-the-ilo/newsroom/news/WCMS_526160/ lang--es/index.htm (consultado 10/2020).

http://www.bancomundial.org/es/publication/human-capital (consultado $1 / 5 / 2019)$.

https://www.oecd.org/centrodemexico/laocde (consultado 1/5/2019)

\section{Referencias}

Álvarez Leguizamón, S. (noviembre 2001). "Los cambios operados en las concepciones de gestión de programas sociales a partir del financiamiento internacional", VI Congreso Internacional del CLAD sobre la Reforma del Estado y de la Administración Pública. Buenos Aires, Argentina, 5-9 de noviembre de 2001. Ponencia.

Babb, S. (2009). Behind the development banks. Washington politics, world poverty, and the wealth of nations. The University of Chicago Press.

Babb, S. (2013). The Washington Consensus as transnational policy paradigm: Its origins, trajectory and likely successor. International Political Economy, 20(2), 268-297.

Bonnet, A. (2001). La globalización y las crisis latinoamericanas. Revista Bajo el Volcán 2(3), 13-31.

Becker, G. y Tomes, N. (1994). "Human Capital and Rise and Fall of Families". En G. Becker y N. Tomes, Human Capital: a Theoretical and Empirical Analysis with Special Reference to Education. The University of Chicago Press. ( $3^{\circ}$ edición).

Cantamutto, F. y Castiglione, L. (2021). ¿Una crisis mundial de deuda?. En G. Roffinelli, A. López Bolaños. y L. Castiglioni (Comps.), Crisis capitalista mundial en tiempos de pandemia. Una mirada desde Nuestra América. Buenos Aires: CLACSO.

Carcedo, F. (2017). El rol del Banco Mundial en la cooperación internacional para la gestión del medioambiente: la cooperación con Argentina en la problemática de la cuenca Matanza-Riachuelo. Revista Integración y Cooperación Internacional, 24, 15-27.

Casaburi, G., Riggirozzi, M. y Tuozzo, M. (2000). "BMDs-sociedad civil: Luces y sombras de una nueva relación". En D. Tussie Luces y sombras de una relación. El Banco Interamericano de Desarrollo, el Banco Mundial y la sociedad civil. Buenos Aires: FLACSO-Temas Grupo Editorial. 
Chiara, M. y Di Virgilio, M. (2005). Gestión social y municipios: De los escritorios del Banco Mundial a los barrios del Gran Buenos Aires. Buenos Aires: Prometeo Libros- Universidad Nacional de General Sarmiento.

Cleaver, H. (1989). Close the IMF, abolish debt and end development: a class analysis of the international debt crisis. Capital \& Class, 39, 17-50.

Coraggio, J. (1994). "Las nuevas políticas sociales: el papel de las agencias multilaterales". Ponencia presentada en el Seminario-Taller Internacional sobre "Estrategias de lucha contra la pobreza y el desempleo estructural: Diseño y gestión de políticas sociales en un marco de globalización económica e integración regional”, CEUR y el Instituto de Geografía de la UBA, Buenos Aires, Argentina. 26-28.

Corbalán, A. (2002). Banco Mundial. Intervención y disciplinamiento. El caso argentino, enseñanzas para América Latina. Buenos Aires: Biblos. Caps. I, II y IV.

Fernández, E. (2021a). La internacionalización de los Programas de Transferencias Monetarias Condicionadas (1995-2008): una revisión por América Latina, África Subsahariana y el Sudeste Asiático. E-latina, 20, 23-47.

Fernández, E. (2021b). La internacionalización de la política asistencial argentina: la política del Banco Mundial hacia la asistencia social (2002-2018). Revista de Relaciones Internacionales. En prensa.

Fiszbein, A. y Schady, N. (2009). Conditional Cash Transfers. Reducing Present and Future Poverty. Washington: The International Bank for Reconstruction and Development.

Garland, D. (2018). Castigar y asistir. Una historia de las estrategias penales y sociales del siglo XX. Buenos Aires: Siglo XXI. Cap. 1.

Grassi, E., Hintze, S. y Neufeld, M. (1994). Políticas sociales, crisis y ajuste estructural: un análisis del sistema educativo, de obras sociales y las politicas alimentarias. Buenos Aires: Espacio.

Hall, P y Taylor, R. (1996). Political Science and the Three New Institutionalisms. Political Studies, XLIV, 936-957.

Hall, A. (2007). Social Policies in the World Bank. Paradigms and Challenges. Global Social Policy, 7, 151-175.

Holloway, J. (1994). Se abre el abismo. Surgimiento y caída del keynesianismo. En J. Holloway, Marxismo, Estado y Capital. La crisis como expresión del poder del trabajo. Buenos Aires: Editorial Tierra del Fuego. 37-75.

Lindahl, M., Palme, M., Sandgren-Massih, S., Sjögren, A. (2014). A test of de BeckerTomes Model of Human Capital Transmission Using Microdata on Four Generations. Journal of Human Capital, 8(1), 80-96.

Lichtensztejn, S. (2012). El Fondo Monetario Internacional y el Banco Mundial. Sus relaciones con el poder financiero. Economía UNAM, 9, 14-28.

Malaluan, J. y Guttal, S. (2003). Poverty Reduction Strategy Papers: A Poor Package for Poverty Reduction, 1-22. Recuperado de http://www.cadtm.org/IMG/pdf/prsp. pdf.

Mallardi, M. (2016). Fundamentos y génesis de la cuestión social: acumulación originaria, patriarcado y conquista. Serviço Social \& Sociedade, 127, 533-554.

Marx, C. (2009). El Capital. Tomo I, Vol. I. Buenos Aires: Siglo XXI Editores.

Mendes Pereira, J. (2010). O Banco Mundial e a construção político-intelectual do "combate à pobreza. Topo, 11(21), 260-282.

Mendes Pereira, J. (2012). Servirse de los pobres: El Banco Mundial y la guerra contra la pobreza. Estudios Criticos del Desarrollo, 2(2),107-146. 
Mendes Pereira, J. (2017). Metamorfoses da política de ajuste estrutural do Banco Mundial (1980-2014). Sociologias, 19(44), 390-422.

Murillo, S. (2006). Banco Mundial. Estado, mercado y sujetos en las nuevas estrategias frente a la cuestión social. Buenos Aires: Ediciones CCC.

Panitch, L. (1998). The State in a Changing World" Social-Democratizing Global Capitalism?. Monthly Review, 50(5), 11-22.

Ruckert, A. (2010). The forgotten dimension of social reproduction: the World Bank and the poverty reduction strategy paradigm. Review of International Political Economy, 17(5), 816-839.

Piva, A. (2020). Una lectura política de la internacionalización del capital. Algunas hipótesis sobre la actual fase de la internacionalización del capital y el Estado nacional de competencia, en V. Ciolli, F. Naspleda, y R. Bernado (Comps.), La dimensión inevitable: estudios sobre la internacionalización del Estado y del capital desde Argentina. (pp. 13-41). Bernal: UNQ.

Toussaint, E. (2004). Banco Mundial, el golpe de Estado permanente. La agenda oculta del Consenso de Washington. París: CADTM.

Vakis, R., Rigolini, J. y Lucchetti, L. (2015). Los olvidados. Pobreza crónica en América Latina y el Caribe. World Bank.

Wade, R. (2001). Showdown at the World Bank. New Left Review 7, 124-137.

\section{Notas}

1 Agradezco a quienes evaluaron la versión previa de este artículo por sus valiosos comentarios que contribuyeron a la mejora del mismo, eximiéndoles de los posibles errores que aún pueda contener.

2 Este último dato no considerando los préstamos que otorga el Banco de Inversión Europeo dentro de Europa; si se consideran, el Banco de Inversión Europeo llega a más de 500000000000 .

3 El objeto de nuestro análisis es lo que estrictamente se suele denominar como Banco Mundial, conformado por dos organizaciones: el BIRF (Banco Internacional para la Reconstrucción y Fomento) y la AIF (Asociación Internacional de Fomento). Es decir, no analizaremos el Grupo Banco Mundial, que integra, además de las dos organizaciones mencionadas, a otras tres: la CFI (Corporación Financiera Internacional), que ofrece créditos y asistencia técnica dirigida a estimular la inversión del sector privado en los países en desarrollo; el OMGI (Organismo Multilateral de Garantía de Inversiones), que ofrece a los inversionistas garantías contra pérdidas causadas por riesgos no comerciales en países en desarrollo; y el CIADI (Centro Internacional para el Arreglo de Diferencias relativas de inversión), un organismo de conciliación y arbitraje de las disputas de inversión.

4 En sus orígenes el mayor peso en la orientación de los préstamos se ubicó en el sector de infraestructura -eléctrica, de comunicación (carreteras, ferrocarriles)- y el sector de la producción - predominantemente hacia el área de la agricultura: mecanización, promoción de cultivos de exportación, etc.-. Luego, hacia el año 1962, se incorporan préstamos destinados al sector de salud, de educación y a infraestructura urbana, como también sistemas de distribución de agua potable y saneamiento de aguas residuales (Toussaint, 2004). Por otra parte, esta política durante los cincuenta y los sesenta se articuló a un enfoque de desarrollo que "concebía ese proceso como la transformación de una economía tradicional, eminentemente agrícola, en otra moderna de tipo industrial que requería como requisito fundamental mejorar las condiciones de su infraestructura. Conforme la concepción del Banco sobre el crecimiento económico, se establecían como relaciones fundamentales, las que, cuantitativamente, se entablaban entre la formación del ahorro y la inversión, por un lado, y la de este binomio con el circuito circular asociado a la expansión productiva” (Lichtensztejn, 2012: 17). 
5 Sobre la revisión neoliberal del neoliberalismo, Cfr. Panitch (1998) y Wade (2001).

6 Específicamente, se plantea "la fijación de prioridades estratégicas; la reorganización de provisión de servicios públicos; el desplazamiento hacia sistemas de responsabilidad basados en el desempeño (o basados en los resultados) y el respaldo de tales sistemas mediante reformas de gestión financiera y de personal” (Banco Mundial, 1998: 152). Sobre el neo-institucionalismo Cfr. Hall \& Taylor (1996).

7 Las contribuciones básicas son las donaciones que realizan los países (en este caso los miembros de la CAD) a organismos multilaterales, o sea, sin controlar el destino final de los fondos donados.

8 Una aclaración metodológica: el cálculo es entre los desembolsos brutos (sin descontar reembolsos) del BM y los desembolsos brutos de ODA. Estos últimos contienen un porcentaje de desembolsos brutos del BM (aquellos que cumplen con el requisito para ser clasificados como ayuda oficial), por lo que las cifras arrojadas del cálculo sobreestiman en parte el peso de la ODA. O, lo que es lo mismo, subestiman en parte el peso del financiamiento del BM en el conjunto del financiamiento oficial mundial. Recordemos que, según la definición tradicional del CAD, la ODA contiene donaciones y préstamos realizados a un tipo de interés por debajo del mercado y con un elemento de donación del 25\% como mínimo. Este último punto cambió en 2014. Según el tipo de país destinatario de la ayuda reembolsable, el elemento de donación exigido varía entre un $10 \%$ y un $45 \%$. En cuanto a la tasa de descuento de referencia, tradicionalmente se consideraba el $10 \%$ pero tras la reforma se sitúa entre el $6 \%$ y el $9 \%$ (dependiendo nuevamente del país socio). La reforma entra definitivamente en vigor en 2019 (para la contabilización de los flujos de 2018). Visto en: http://www.realida dayuda.org/ (consultado 12/2019).

9 La incorporación, a esta altura de la trayectoria del BM, del concepto de pobreza contrasta con el concepto de "necesidades básicas" presente desde los comienzos del Banco Mundial.

10 La continuidad ininterrumpida del eje en el llamado "combate a la pobreza" en la política del Banco es materia de discusión. Hay autores que señalan la discontinuidad de este eje de intervención del BM durante los ochenta e incluso los noventa, por ejemplo: Babb (2013) y Hall (2007). En este trabajo nos ceñimos a la posición de Mendes Pereira (2012), quien plantea más una continuidad que una discontinuidad en este punto.

11 Sobre el concepto y la práctica de la participación de la sociedad civil en los lineamientos no solo de BM sino también del BID, Cfr. Casaburi, Riggirozzi y Tuozzo. (2000).

12 Continúa: "El ritmo y el diseño de la descentralización condicionarán las consecuencias que ésta pueda tener en la eficacia, la obligación de rendir cuentas, la participación y, en definitiva, la reducción de la pobreza" (BM, 2001:106).

13 “Cómo puede la intervención pública capacitar a los pobres para que amplíen sus activos, y cómo puede resolver las desigualdades en ese sentido? Hay tres formas complementarias: la utilización del poder estatal para redistribuir los recursos, sobre todo en los servicios que crean activos, como la educación la salud y la infraestructura; la reforma de las políticas y las instituciones para garantizar una prestación eficaz de los servicios, y la implicación de los hogares pobres en las decisiones sobre la variedad, funcionamiento, supervisión y evaluación de los programas y servicios que incrementan sus activos." (BM, 2001:79). Por otro lado: "Para conseguir que el gasto público redunde en mayor beneficio de los necesitados, habrá que reducir el gasto militar y las subvenciones a quienes no las necesitan. La privatización de las empresas públicas ineficientes y con pérdidas libera recursos que podrían destinarse a atender las necesidades de los pobres" (BM, 2001:82).

14 "Los pobres necesitan poder opinar directamente sobre las intervenciones que afectan sus vidas cotidianas, así como tener la capacidad de organizarse y votar. Es necesario actuar para derribar las barreras — legales, políticas, administrativas o sociales-que perjudican a grupos particulares e incrementar los activos de los pobres para impedir su exclusión del mercado" (BM, 2001: 115).

15 Datos extraídos de: https://www.imf.org/external/np/jsa/index.aspx\#U (consultado 10/2020) 
16 Datos extraídos de: https://www.bancomundial.org/es/results/2013/04/11/global-f ood-crisis-response-program-results-profile (consultado 10/2020).

17 En América Latina y el Caribe de un total de 28 programas de transferencias monetarias que se implementan en la actualidad en 20 países de la región, diez recibieron o reciben financiamiento del BM y/o del BID (de esos diez, solo en uno no está presente el BM). Esto significa que la mitad de los países latinoamericanos o caribeños que implementa algún tipo de programa de transferencia monetaria recibe o recibió apoyo técnicofinanciero del BM o del BID.

18 El Banco organizó la Conferencia Internacional sobre Programas de Transferencias Condicionadas en mayo de 2002 en Puebla, en abril de 2004 en San Pablo y en junio de 2006 en Estambul. También, el principal documento internacional sobre los PTMC sale del seno de este organismo, nos referimos a: Las transferencias condicionadas reduciendo pobreza hoy y en el futuro, elaborado por Fiszbein y Schady y publicado por el Banco en el año 2009.

19 https://www.ilo.org/global/about-the-ilo/newsroom/news/WCMS_526160/lang-es/index.htm (consultado 10/2020).

20 Este marco de acuerdo con 150 países es la continuación de los Objetivos de Desarrollo del Milenio (ODM) convenidos en el año 2000, que fijaron metas mundiales para el período 2000-2015.

21 Con respecto a la resiliencia dice: “(...) para generar resiliencia frente a las crisis y las amenazas - aunque continuamos desarrollando infraestructura climáticamente inteligente y mejorando los sistemas de respuesta-, necesitamos instrumentos financieros novedosos para ayudar a los países pobres a hacer lo que las naciones ricas han venido haciendo durante mucho tiempo: compartir los riesgos de las crisis con los mercados de capitales mundiales".

22 Cfr. http://www.bancomundial.org/es/publication/human-capital

23 Los olvidados. Pobreza crónica en América Latina y el Caribe (2015), elaborado por Vakis, Rigolini y Lucchetti.

24 Debemos advertir que en el cálculo dejamos afuera al sector de Agricultura porque no corresponde a un área de la política social. Sin embargo, hay que observar que en este sector se contienen préstamos destinados hacia pequeños agricultores, que están planteados por el mismo Banco como parte de la estrategia de reducción de la pobreza.

25 Trabajamos esto en Fernández (2021b). 